\title{
ON THE NUMBER OF LIMIT CYCLES FOR A GENERALIZATION OF LIÉNARD POLYNOMIAL DIFFERENTIAL SYSTEMS
}

\author{
JAUME LLIBRE \\ Departament de Matemàtiques \\ Universitat Autònoma de Barcelona, 08193, Bellaterra, Barcelona, \\ Catalonia, Spain,e-mail: jllibre@mat.uab.cat \\ CLAUDIA VALLS \\ Departamento de Matemática \\ Instituto Superior Técnico, 1049-001 Lisboa, Portugal \\ e-mail: cvalls@math.ist.utl.pt
}

February 2, 2012

We study the number of limit cycles of the polynomial differential systems of the form

$$
\dot{x}=y-g_{1}(x), \quad \dot{y}=-x-g_{2}(x)-f(x) y,
$$

where $g_{1}(x)=\varepsilon g_{11}(x)+\varepsilon^{2} g_{12}(x)+\varepsilon^{3} g_{13}(x), g_{2}(x)=$ $\varepsilon g_{21}(x)+\varepsilon^{2} g_{22}(x)+\varepsilon^{3} g_{23}(x)$ and $f(x)=\varepsilon f_{1}(x)+$ $\varepsilon^{2} f_{2}(x)+\varepsilon^{3} f_{3}(x)$ where $g_{1 i}, g_{2 i}, f_{2 i}$ have degree $k$, $m$ and $n$ respectively for each $i=1,2,3$, and $\varepsilon$ is a small parameter. Note that when $g_{1}(x)=0$ we obtain the generalized Liénard polynomial differential systems. We provide an upper bound of the maximum number of limit cycles that the previous differential system can have bifurcating from the periodic orbits of the linear center $\dot{x}=y, \dot{y}=-x$ using the averaging theory of third order.

\section{Introduction and statement of the main results}

The second part of the 16th Hilbert's problem wants to find an upper bound for the maximum number of limit cycles that a polynomial vector field of a fixed degree can have. In this paper we will try to give a partial answer to this problem for the class of polynomial differential systems

$$
\dot{x}=y-g_{1}(x), \quad \dot{y}=-x-g_{2}(x)-f(x) y,
$$

where

$$
\begin{aligned}
g_{1}(x) & =\varepsilon g_{11}(x)+\varepsilon^{2} g_{12}(x)+\varepsilon^{3} g_{13}(x), \\
g_{2}(x) & =\varepsilon g_{21}(x)+\varepsilon^{2} g_{22}(x)+\varepsilon^{3} g_{23}(x), \\
f(x) & =\varepsilon f_{1}(x)+\varepsilon^{2} f_{2}(x)+\varepsilon^{3} f_{3}(x),
\end{aligned}
$$

where $g_{1 i}, g_{2 i}, f_{i}$ have degree $k, m$ and $n$ respectively for each $i=1,2,3$, and $\varepsilon$ is a small parameter. When $g_{1}(x)=0$ the differential system (1) coincides with the generalized Liénard polynomial differential systems. The classical Liénard polynomial differential systems are

$$
\dot{x}=y, \quad \dot{y}=-x-f(x) y,
$$

where $f(x)$ is a polynomial in the variable $x$ of degree $n$. For these systems [Lins et al., 1977] stated the conjecture that if $f(x)$ has degree $n \geq 1$ then 
system (2) has at most $[n / 2]$ limit cycles. Here $[x]$ denotes the integer part function of $x \in \mathbb{R}$. They proved this conjecture for $n=1,2$. The conjecture for $n=3$ has been proved recently by [Li \& Llibre, 2012]. For $n \geq 5$ the conjecture is not true, see [De Maesschalck \& Dumortier, 2011] and [Dumortier et al., 2007]. So it remains to know if the conjecture is true or not for $n=4$.

Many of the results on the limit cycles of polynomial differential systems have been obtained by considering limit cycles which bifurcate from a single degenerate singular point (i.e., from a Hopf bifurcation), that are called small amplitude limit cycles, see for instance [Lloyd, 1988]. There are partial results concerning the maximum number of small amplitude limit cycles for Liénard polynomial differential systems. The number of small amplitude limit cycles gives a lower bound for the maximum number of limit cycles that a polynomial differential system can have.

There are many results concerning the existence of small amplitude limit cycles for the following generalization of the classical Liénard polynomial differential system (2)

$$
\dot{x}=y, \quad \dot{y}=-g(x)-f(x) y,
$$

where $g(x)$ and $f(x)$ are polynomials in the variable $x$ of degrees $m$ and $n$, respectively. We denote by $H(m, n)$ the maximum number of limit cycles that systems (3) can have. This number is usually called the Hilbert number for systems (3).

(i) [Liénard, 1928] proved that if $m=1$ and $F(x)=\int_{0}^{x} f(s) d s$ is a continuous odd function, which has a unique root at $x=a$ and is monotone increasing for $x \geq a$, then equation (3) has a unique limit cycle.

(ii) [Rychkov, 1975] proved that if $m=1$ and $F(x)$ is an odd polynomial of degree five, then equation (3) has at most two limit cycles.

(iii) [Lins et al., 1977] proved that $H(1,1)=0$ and $H(1,2)=1$.

(iv) [Coppel, 1998] proved that $H(2,1)=1$.

(v) [Dumortier \& Rousseau, 1990] and [Dumortier \& Li, 1996] shown that $H(3,1)=1$.

(vi) [Dumortier \& Li, 1997] proved that $H(2,2)=$ 1. (vii) [Lloyd \& Lynch, 1988] proved that $H(1,3)=$ 1.

Up to now and as far as we know only for these four cases ((iii)-(vii)) the Hilbert number for systems (3) has been determined.

The maximum number of small amplitude limit cycles for systems (3) is denoted by $\hat{H}(m, n)$. [Blows \& Lloyd, 1984], [Lloyd \& Lynch, 1988] and [Lynch, 1995] have used inductive arguments in order to prove the following results.

(I) If $g$ is odd then $\hat{H}(m, n)=[n / 2]$.

(II) If $f$ is even then $\hat{H}(m, n)=n$, whatever $g$ is.

(III) If $f$ is odd then $\hat{H}(m, 2 n+1)=[(m-2) / 2]+n$.

(IV) If $g(x)=x+g_{e}(x)$, where $g_{e}$ is even then $\hat{H}(2 m, 2)=m$.

[Christopher \& Lynch, 1999], [Lynch, 1998], [Lynch, 1999], [Lynch \& Christopher, 1999] have developed a new algebraic method for determining the Liapunov quantities of systems (3) and proved some other bounds for $\hat{H}(m, n)$ for different $m$ and $n$.

(V) $\hat{H}(m, 2)=[(2 m+1) / 3]$.

(VI) $\hat{H}(2, n)=[(2 n+1) / 3]$.

(VII) $\hat{H}(m, 3)=2[(3 m+2) / 8]$ for all $1<m \leq 50$.

(VIII) $\hat{H}(3, n)=2[(3 n+2) / 8]$ for all $1<m \leq 50$.

(IX) $\hat{H}(4, k)=\hat{H}(k, 4), k=6,7,8,9$ and $\hat{H}(5,6)=$ $\hat{H}(6,5)$.

[Gasull \& Torregrosa, 1998] obtained upper bounds for $\hat{H}(7,6), \hat{H}(6,7), \hat{H}(7,7)$ and $\hat{H}(4,20)$.

[Yu \& Han, 2006] give some accurate values of $\hat{H}(m, n)=\hat{H}(n, m)$, for $n=4, m=10,11,12,13$; $n=5, m=6,7,8,9 ; n=6, m=5,6$, see also [Llibre et al., 2009] for a table with all the specific values.

[Llibre et al., 2009] compute the maximum number of limit cycles $\tilde{H}_{k}(m, n)$ of systems (3) which bifurcate from the periodic orbits of the linear center $\dot{x}=y, \dot{y}=-x$, using the averaging theory of order $k$, for $k=1,2,3$. 
In [Llibre \& Valls, 2011] the authors studied using the averaging theory of first and second order the more general system

$$
\begin{aligned}
\dot{x}= & y-\varepsilon\left(g_{11}(x)+f_{11}(x) y\right)- \\
& \varepsilon^{2}\left(g_{12}(x)+f_{12}(x) y\right), \\
\dot{y}= & -x-\varepsilon\left(g_{21}(x)+f_{21}(x) y\right)- \\
& \varepsilon^{2}\left(g_{22}(x)+f_{22}(x) y\right),
\end{aligned}
$$

where $g_{1 i}, f_{1 i}, g_{2 i}, f_{2 i}$ have degree $k, l, m$ and $n$ respectively for each $i=1,2$, and $\varepsilon$ is a small parameter. Using the averaging method of first and second order they proved the following result.

Theorem 1.1. For $|\varepsilon|$ sufficiently small the maximum number of limit cycles of the generalized Liénard polynomial differential systems (4) bifurcating from the periodic orbits of the linear center $\dot{x}=y, \dot{y}=-x$ using the averaging theory of second order is:

$$
\begin{aligned}
\max & \{\mu+[(m-1) / 2], \mu+[k / 2], \\
& {[(n-1) / 2]+[m / 2], } \\
& {[l / 2]+[m / 2]-1, } \\
& {[(n-1) / 2]+[(k-1) / 2]+1, } \\
& {[l / 2]+[(k-1) / 2]\}, }
\end{aligned}
$$

with $\mu=\min \{[n / 2],[(l-1) / 2]\}$.

[Alavez-Ramirez et al., 2012] studied system (1) with $g_{13}(x)=g_{23}(x)=f_{23}(x)=0$ and they proved the following result.

Theorem 1.2. For $|\varepsilon|$ sufficiently small the maximum number of limit cycles of the generalized Liénard polynomial differential systems (4) with $f_{11}(x)=f_{12}(x)=0$ bifurcating from the periodic orbits of the linear center $\dot{x}=y, \dot{y}=-x$ using the averaging theory of third order is

$$
\lambda_{0}=\left[\frac{1}{2}(\max \{O(m+n), E(k+m)-1\}-1)\right],
$$

where $O(l)$ is the largest odd integer $\leq l$, and $E(l)$ is the largest even integer $\leq l$.

In the present paper we study system (1), i.e. we extend the results of [Alavez-Ramirez et al., 2012] because in [Alavez-Ramirez et al., 2012] first $g_{13}(x)=g_{23}(x)=f_{3}(x)=0$, and additionally the study of the limit cycles coming from averaging of second and third order is made under some restrictive conditions. Using the averaging method of third order we will show our main result:

Theorem 1.3. For $|\varepsilon|$ sufficiently small the maximum number of limit cycles of the generalized Liénard polynomial differential systems (1) bifurcating from the periodic orbits of the linear center $\dot{x}=y, \dot{y}=-x$ using the averaging theory of third order is $\lambda_{1}$ equal to

$$
\begin{aligned}
\max & \{2 m+2[k / 2]-2, \\
& m+2[(k-1) / 2], \\
& m+2[(n-1) / 2]+2[m / 2]+2, \\
& 4[(n-1) / 2]+2[(m-1) / 2]+1\} .
\end{aligned}
$$

Note that $\lambda_{0}<\lambda_{1}$. The proof of Theorem 1.3 is given in Section 3 .

The results that we shall use from the averaging theory of third order for computing limit cycles are presented in Section 2.

\section{The averaging theory of third order}

The averaging theory for studying specifically limit cycles up to third order in $\varepsilon$ was developed in [Buică \& J. Llibre, 2004]. It is summarized as follows.

Consider the differential system

$$
\begin{aligned}
\dot{x}= & \varepsilon F_{1}(t, x)+\varepsilon^{2} F_{2}(t, x)+ \\
& \varepsilon^{3} F_{3}(t, x)+\varepsilon^{4} R(t, x, \varepsilon),
\end{aligned}
$$

where $F_{1}, F_{2}, F_{3}: \mathbb{R} \times D \rightarrow \mathbb{R}, R: \mathbb{R} \times D \times\left(-\varepsilon_{f}, \varepsilon_{f}\right) \rightarrow$ $\mathbb{R}$ are continuous functions, $T$-periodic in the first variable, and $D$ is an open subset of $\mathbb{R}^{n}$. Assume that the following conditions hold.

(i) $F_{1}(t, \cdot) \subset C^{2}(D), F_{2}(t, \cdot) \subset C^{1}(D)$ for all $t \in \mathbb{R}, F_{1}, F_{2}, F_{3}, R$ are locally Lipschitz with respect to $x$, and $R$ is twice differentiable with respect to $\varepsilon$.

We define $F_{k 0}: D \rightarrow \mathbb{R}$ for $k=1,2$ as

$$
\begin{aligned}
F_{10}(z)= & \frac{1}{T} \int_{0}^{T} F_{1}(s, z) d s \\
F_{20}(z)= & \frac{1}{T} \int_{0}^{T}\left[D_{z} F_{1}(s, z) y_{1}(s, z)+\right. \\
& \left.F_{2}(s, z)\right] d s
\end{aligned}
$$




$$
\begin{aligned}
& F_{30}(z)=\frac{1}{T} \int_{0}^{T}\left(\frac{1}{2} \frac{\partial^{2} F_{1}}{\partial z^{2}}(s, z) y_{1}(s, z)^{2}+\right. \\
& \frac{1}{2} \frac{\partial F_{1}}{\partial z}(s, z) y_{2}(s, z)+ \\
&\left.\frac{\partial F_{2}}{\partial z}(s, z) y_{1}(s, z)+F_{3}(s, z)\right) d s,
\end{aligned}
$$

where

$$
\begin{gathered}
y_{1}(s, z)=\int_{0}^{s} F_{1}(t, z) d t \\
y_{2}(s, z)=2 \int_{0}^{s}\left(\frac{\partial F_{1}}{\partial z}(t, z) \int_{0}^{t} F_{1}(r, z) d r\right. \\
\left.\quad+F_{2}(t, z)\right) d t
\end{gathered}
$$

(ii) For $V \subset D$ an open and bounded set and for each $\varepsilon \in\left(-\varepsilon_{f}, \varepsilon_{f}\right) \backslash\{0\}$, there exists $a_{\varepsilon} \in V$ such that $F_{10}\left(a_{\varepsilon}\right)+\varepsilon F_{20}\left(a_{\varepsilon}\right)+\varepsilon^{2} F_{30}\left(a_{\varepsilon}\right)=0$ and $d_{B}\left(F_{10}+\varepsilon F_{20}+\varepsilon^{2} F_{30}, V, a_{\varepsilon}\right) \neq 0$.

Then for $|\varepsilon|>0$ sufficiently small there exists a $T$-periodic solution $\phi(\cdot, \varepsilon)$ of the system such that $\phi\left(0, a_{\varepsilon}\right) \rightarrow a_{\varepsilon}$ when $\varepsilon \rightarrow 0$.

The expression $d_{B}\left(F_{10}+\varepsilon F_{20}+\varepsilon^{2} F_{30}, V, a_{\varepsilon}\right) \neq$ 0 means that the Brouwer degree of the function $F_{10}+\varepsilon F_{20}+\varepsilon^{2} F_{30}: V \rightarrow \mathbb{R}^{n}$ at the fixed point $a_{\varepsilon}$ is not zero. A sufficient condition in order that this inequality is true is that the Jacobian of the function $F_{10}+\varepsilon F_{20}+\varepsilon^{2} F_{30}$ at $a_{\varepsilon}$ is not zero.

If $F_{10}$ is not identically zero, then the zeros of $F_{10}+\varepsilon F_{20}+\varepsilon^{2} F_{30}$ are mainly the zeros of $F_{10}$ for $\varepsilon$ sufficiently small. In this case the previous result provides the averaging theory of first order.

If $F_{10}$ is identically zero and $F_{20}$ is not identically zero, then the zeros of $F_{10}+\varepsilon F_{20}+\varepsilon^{2} F_{30}$ are mainly the zeros of $F_{20}$ for $\varepsilon$ sufficiently small. In this case the previous result provides the averaging theory of second order.

If $F_{10}$ and $F_{20}$ are identically zero and $F_{30}$ is not identically zero, then the zeros of $F_{10}+\varepsilon F_{20}+$ $\varepsilon^{2} F_{30}$ are mainly the zeros of $F_{30}$ for $\varepsilon$ sufficiently small. In this case the previous result provides the averaging theory of third order.

\section{Proof of Theorem 1.3}

We shall need the third order averaging theory to prove Theorem 1.3. We write system (1) in polar coordinates $(r, \theta)$ where

$$
x=r \cos \theta, \quad y=r \sin \theta, \quad r>0 .
$$

If we write

$$
\begin{aligned}
f_{1}(x) & =\sum_{i=0}^{n} a_{i} x^{i}, \\
f_{2}(x) & =\sum_{i=0}^{n} c_{i} x^{i}, \\
f_{3}(x) & =\sum_{i=0}^{n} p_{i} x^{i}, \\
g_{11}(x) & =\sum_{i=0}^{k} b_{i, 1} x^{i}, \\
g_{12}(x) & =\sum_{i=0}^{k} d_{i, 1} x^{i}, \\
g_{13}(x) & =\sum_{i=0}^{k} q_{i, 1} x^{i}, \\
g_{21}(x) & =\sum_{i=0}^{m} b_{i, 2} x^{i}, \\
g_{22}(x) & =\sum_{i=0}^{m} d_{i, 2} x^{i}, \\
g_{23}(x) & =\sum_{i=0}^{m} q_{i, 2} x^{i},
\end{aligned}
$$

then system (1) becomes

$$
\begin{aligned}
\dot{r} & =-\varepsilon\left(A+\varepsilon B+\varepsilon^{2} C\right), \\
\dot{\theta} & =-1-\frac{\varepsilon}{r}\left(A_{1}+\varepsilon B_{1}+\varepsilon^{2} C_{1}\right),
\end{aligned}
$$

where

$$
\begin{aligned}
A= & \sum_{i=0}^{n} a_{i} r^{i+1} \cos ^{i} \theta \sin ^{2} \theta+ \\
& \sum_{i=0}^{m} b_{i, 2} r^{i} \cos ^{i} \theta \sin \theta+ \\
B= & \sum_{i=0}^{k} b_{i, 1} r^{i} \cos ^{i+1} \theta, \\
& \sum_{i=0}^{n=0} c_{i} r^{i+1} d_{i, 2} r^{i} \cos ^{i} \theta \sin \theta+ \\
& \sum_{i=0}^{k} d_{i, 1} r^{i} \cos ^{i+1} \theta,
\end{aligned}
$$




$$
\begin{aligned}
C= & \sum_{i=0}^{n} p_{i} r^{i+1} \cos ^{i} \theta \sin ^{2} \theta+ \\
& \sum_{\substack{m=0 \\
m}} q_{i, 2} r^{i} \cos ^{i} \theta \sin \theta+ \\
& \sum_{i=0}^{k} q_{i, 1} r^{i} \cos ^{i+1} \theta
\end{aligned}
$$

and

$$
\begin{aligned}
A_{1}= & \sum_{i=0}^{n} a_{i} r^{i+1} \cos ^{i+1} \theta \sin \theta+ \\
& \sum_{i=0}^{m} b_{i, 2} r^{i} \cos ^{i+1} \theta- \\
& \sum_{i=0}^{k} b_{i, 1} r^{i} \cos ^{i} \theta \sin \theta \\
B_{1}= & \sum_{i=0}^{n} c_{i} r^{i+1} \cos ^{i+1} \theta \sin \theta+ \\
& \sum_{i=0}^{m} d_{i, 2} r^{i} \cos ^{i+1} \theta- \\
& \sum_{i=0}^{k} d_{i, 1} r^{i} \cos ^{i} \theta \sin \theta \\
C_{1}= & \sum_{i=0}^{n} p_{i} r^{i+1} \cos ^{i+1} \theta \sin \theta+ \\
& \sum_{i=0}^{m} q_{i, 2} r^{i} \cos ^{i+1} \theta- \\
& \sum_{i=0}^{k} q_{i, 1} r^{i} \cos ^{i} \theta \sin \theta
\end{aligned}
$$

Now taking $\theta$ as the new independent variable, system (9) becomes

$$
\frac{d r}{d \theta}=\varepsilon F_{1}(r, \theta)+\varepsilon^{2} F_{2}(r, \theta)+\varepsilon^{3} F_{3}(r, \theta)+O\left(\varepsilon^{4}\right),
$$

where

$$
\begin{aligned}
& F_{1}(r, \theta)=A, \quad F_{2}(r, \theta)=B-\frac{1}{r} A A_{1}, \\
& F_{3}(r, \theta)=C-\frac{1}{r}\left(B A_{1}+A B_{1}\right)+\frac{1}{r^{2}} A_{1}^{2} A .
\end{aligned}
$$

In [Alavez-Ramirez et al., 2012] the authors took the sufficient conditions

$$
b_{2 i+1,1}=0 \quad \text { and } \quad a_{2 j, 2}=0,
$$

for $i=0, \ldots,[(k-1) / 2]$ and $j=0, \ldots,[n / 2]$ to have $F_{10}=0$. Furthermore, using this condition they computed $F_{20}$ and they took

$$
\begin{gathered}
d_{2 i+1,1}=c_{2 i}=0 \begin{array}{l}
\text { for } i=0, \ldots,[(k-1) / 2] \\
\text { and } j=0, \ldots,[n / 2], \text { and }
\end{array} \\
\text { either } b_{i, 1}=a_{j}=0 \text { for } i=0, \ldots, k \text { and } \\
j j=0, \ldots, n, \\
\text { or } b_{i, 2}=0 \text { for } i=0, \ldots, m,
\end{gathered}
$$

thus obtaining an upper bound for the number of limit cycles. However a close look to $F_{20}(r)$ obtained in [Alavez-Ramirez et al., 2012] imply that it is only necessary to take

$$
\begin{aligned}
& \sum_{\substack{i=0 \\
[k / 2]}}^{[(k-1) / 2]} d_{2 i+1,1}+\sum_{i=0}^{[n / 2]} \frac{c_{2 i}}{2 i+1}+ \\
& \sum_{i=0}^{[n / 2]} \sum_{j=0}^{[(n-1) / 2]} C_{i j} b_{2 i, 1} b_{2 j, 2}+ \\
& \sum_{i=0}^{[m / 2]} \sum_{j=0}^{[m / 2} D_{i j} a_{2 i+1} b_{2 j, 2}=0,
\end{aligned}
$$

for some constants $C_{i j}$ and $D_{i j}$.

We shall work only with the necessary conditions (11) and (12), and consequently we improve the results of [Alavez-Ramirez et al., 2012] in two ways. First because we work with this necessary conditions and second because we consider in the differential system (1) terms up to order $\varepsilon^{3}$ while in [Alavez-Ramirez et al., 2012] they consider only up to order $\varepsilon^{2}$.

In order to apply the third order averaging method we need to compute the corresponding function $F_{30}(r)$ that we rewrite as

$$
F_{30}(r)=F_{30}^{1}(r)+F_{30}^{2}(r)+F_{30}^{3}(r)+F_{30}^{4}(r)
$$

with

$$
\begin{aligned}
& F_{30}^{1}(r)=\frac{1}{4 \pi} \int_{0}^{2 \pi} \frac{\partial^{2} F_{1}}{\partial r^{2}}(r, \theta) y_{1}(r, \theta)^{2} d \theta \\
& F_{30}^{2}(r)=\frac{1}{4 \pi} \int_{0}^{2 \pi} \frac{\partial F_{1}}{\partial r}(r, \theta) y_{2}(r, \theta) d \theta \\
& F_{30}^{3}(r)=\frac{1}{2 \pi} \int_{0}^{2 \pi} \frac{\partial F_{2}}{\partial r}(r, \theta) y_{1}(r, \theta) d \theta \\
& F_{30}^{4}(r)=\frac{1}{2 \pi} \int_{0}^{2 \pi} F_{3}(r, \theta) d \theta
\end{aligned}
$$

It was proved in [Alavez-Ramirez et al., 2012] that using the integrals of the Appendix, or in 
[Llibre \& Valls, 2011] that $y_{1}=y_{1}(\theta, r)$ is equal to

$$
\begin{aligned}
& \sum_{i=0}^{[(n-1) / 2]} a_{2 i+1} r^{2 i+2} \sum_{s=0}^{i+1} \tilde{\gamma}_{i, s} \sin ((2 s+1) \theta)+ \\
& \sum_{\substack{i=0 \\
[k / 2]}}^{b_{i, 2}} i+1 \\
& \sum_{i=0}^{i} b_{2 i, 1} r^{2 i} \sum_{s=0}^{i} \gamma_{i, s} \sin ((2 s+1) \theta),
\end{aligned}
$$

where

$$
\tilde{\gamma}_{i, l}= \begin{cases}\gamma_{i, l}-\gamma_{i+1, l}, & 0 \leq l \leq i \\ -\gamma_{i+1, i+1}, & l=i+1\end{cases}
$$

and $F_{1}(\theta, r)$ is

$$
\begin{aligned}
& \sum_{i=0}^{[(n-1) / 2]} a_{2 i+1} r^{2 i+2} \cos ^{2 i+1} \theta \sin ^{2} \theta+ \\
& \sum_{\substack{\left.i=0 \\
m^{2} / 2\right]}}^{[k / 2]} b_{i, 2} r^{i} \cos ^{i} \theta \sin \theta+ \\
& \sum_{i=0} b_{2 i, 1} r^{2 i} \cos ^{2 i+1} \theta,
\end{aligned}
$$

finally $\frac{\partial}{\partial r} F_{1}(\theta, r)$ is

$$
\begin{aligned}
& \sum_{i=0}^{[(n-1) / 2]}(2 i+2) a_{2 i+1} r^{2 i+1} \cos ^{2 i+1} \theta \sin ^{2} \theta+ \\
& \sum_{i=0}^{m^{2}} i b_{i, 2} r^{i-1} \cos ^{i} \theta \sin \theta+ \\
& \sum_{i=0}^{[k / 2]} 2 i b_{2 i, 1} r^{2 i-1} \cos ^{2 i+1} \theta .
\end{aligned}
$$

We also note that $F_{2}(r, \theta)$ is equal to

$$
\begin{aligned}
& \sum_{i=0}^{n} c_{i} r^{i+1} \cos ^{i} \theta\left(1-\cos ^{2} \theta\right)+ \\
& \sum_{i=0}^{m} d_{i, 2} r^{i} \cos ^{i} \theta \sin \theta+ \\
& \sum_{i=0}^{k} d_{i, 1} r^{i} \cos ^{i+1} \theta- \\
& \sum_{i=0}^{[(n-1) / 2]} \sum_{j=0}^{[(n-1) / 2]} a_{2 i+1} a_{2 j+1} r^{2 i+2 j+3} \cos ^{2 i+2 j+3} . \\
& 2 \sum_{i=0}^{[(n-1) / 2]} \sum_{j=0}^{m} a_{2 i+1} b_{j, 2} r^{2 i+j+1} \cos ^{2 i+j+2} \theta . \\
& \left(1-\cos ^{2} \theta\right)+
\end{aligned}
$$

$$
\begin{aligned}
& \sum_{i=0}^{[(n-1) / 2]} \sum_{j=0}^{[k / 2]} a_{2 i+1} b_{2 j, 1} r^{2 i+2 j+1} \cos ^{2 i+2 j+1} \theta \\
& \sum_{i=0}^{m} \sum_{j=0}^{m} b_{i, 2} b_{j, 2} r^{i+j-1} \cos ^{i+j+1} \theta \sin \theta+ \\
& \sum_{i=0}^{m} \sum_{j=0}^{[k / 2]} b_{i, 2} b_{2 j, 1} r^{i+2 j-1} \cos ^{i+2 j} \theta\left(1-2 \cos ^{2} \theta\right)+ \\
& \sum_{i=0}^{[k / 2]} \sum_{j=0}^{[k / 2]} b_{2 i, 1} b_{2 j, 1} r^{2 i+2 j-1} \cos ^{2 i+2 j+1} \theta \sin \theta .
\end{aligned}
$$

The proof of Theorem 1.3 will be a direct consequence of the next auxiliary lemmas.

Lemma 3.1. The integral $F_{30}^{1}(r)$ is a polynomial in the variable $r$ of degree

$$
\lambda_{2}=2(m+\max \{[(n-1) / 2],[k / 2]-1\}) .
$$

(For an explicit expression of the polynomial $F_{30}^{1}(r)$ we refer the reader to the proof of Lemma 3.1).

Proof. We first note that $\frac{\partial^{2}}{\partial r^{2}} F_{1}(\theta, r)$ is equal to

$$
\begin{aligned}
& \sum_{i=0}^{[(n-1) / 2]}(2 i+2)(2 i+1) a_{2 i+1} r^{2 i} \\
& \cos ^{2 i+1} \theta\left(1-\cos ^{2} \theta\right)+ \\
& \sum_{\substack{i=0 \\
[k / 2]}}^{m} i(i-1) b_{i, 2} r^{i-2} \cos ^{i} \theta \sin \theta+ \\
& \sum_{i=0}^{[k} 2 i(2 i-1) b_{2 i, 1} r^{2 i-2} \cos ^{2 i+1} \theta,
\end{aligned}
$$

and $y_{1}(r, \theta)^{2}$ is equal to

$$
\begin{aligned}
& \sum_{i=0}^{[(n-1) / 2]} \sum_{j=0}^{[(n-1) / 2]} a_{2 i+1} a_{2 j+1} r^{2 i+2 j+4} . \\
& \sum_{s=0}^{i+1} \sum_{r=0}^{j+1} \tilde{\gamma}_{i, s} \tilde{\gamma}_{j, r} \sin ((2 s+1) \theta) \sin ((2 r+1) \theta)+
\end{aligned}
$$$$
2 \sum_{i=0}^{[(n-1) / 2]} \sum_{j=0}^{m} a_{2 i+1} \frac{b_{j, 2}}{j+1} r^{2 i+j+2} .
$$$$
\sum_{s=0}^{i+1} \tilde{\gamma}_{i, s}\left(1-\cos ^{j+1} \theta\right) \sin ((2 s+1) \theta)+
$$ 


$$
\begin{aligned}
& 2 \sum_{i=0}^{[(n-1) / 2]} \sum_{j=0}^{[k / 2]} a_{2 i+1} b_{2 j, 1} r^{2 i+2 j+2} . \\
& \sum_{s=0}^{i+1} \sum_{r=0}^{j} \tilde{\gamma}_{i, s} \gamma_{j, r} \sin ((2 s+1) \theta) \sin ((2 r+1) \theta)+ \\
& \sum_{i=0}^{m} \sum_{j=0}^{m} \frac{b_{i, 2} b_{j, 2} r^{i+j}}{(i+1)(j+1)}\left(1-\cos ^{i+1} \theta\right)\left(1-\cos ^{j+1} \theta\right)+ \\
& 2 \sum_{i=0}^{m} \sum_{j=0}^{[k / 2]} \frac{b_{i, 2} b_{2 j, 1}}{(i+1)} r^{i+2 j} \sum_{r=0}^{j} \gamma_{j, r}\left(1-\cos ^{i+1} \theta\right) . \\
& \sin ((2 r+1) \theta)+\sum_{i=0}^{[k / 2]} \sum_{j=0}^{[k / 2]} b_{2 i, 1} b_{2 j, 1} r^{2 i+2 j .} . \\
& \sum_{s=0}^{i} \sum_{r=0}^{j} \gamma_{i, s} \gamma_{j, r} \sin ((2 s+1) \theta) \sin ((2 r+1) \theta) .
\end{aligned}
$$

Hence, using the formulae in the appendix and the explicit formula of $F_{30}^{1}(r)$ given in (13) we obtain that $F_{30}^{1}(r)$ is equal to

$$
\begin{aligned}
& \sum_{t=0}^{[(n-1) / 2]} \sum_{i=0}^{m} \sum_{j=0}^{m} \delta_{1} a_{2 t+1} b_{i, 2} b_{j, 2} r^{2 t+i+j}+ \\
& \sum_{t=0}^{[m / 2]} \sum_{i=0}^{[(n-1) / 2]} \sum_{j=0}^{m} \delta_{2} b_{2 t, 2} a_{2 i+1} b_{j, 2} r^{2 t+2 i+j}+ \\
& \sum_{t=0}^{[(m-1) / 2]} \sum_{i=0}^{[(n-1) / 2]} \sum_{j=0}^{[m / 2]} \delta_{3} b_{2 t+1,2} a_{2 i+1} b_{2 j, 2} r^{2 t+2 i+2 j+1}+
\end{aligned}
$$$$
\sum_{t=0}^{[m / 2]} \sum_{i=0}^{m} \sum_{j=0}^{[k / 2]} \delta_{4} b_{2 t, 2} b_{i, 2} b_{2 j, 1} r^{2 t+i+2 j-2}+
$$$$
[(m-1) / 2][m / 2][k / 2]
$$$$
\sum_{t=0} \sum_{i=0}^{m} \sum_{j=0}^{k} \delta_{5} b_{2 t+1,2} b_{2 i, 2} b_{2 j, 1} r^{2 t+2 i+2 j-1}+
$$$$
\sum_{t=0}^{[k / 2]} \sum_{i=0}^{m} \sum_{j=0}^{m} \delta_{6} b_{2 t, 1} b_{i, 2} b_{j, 2} r^{2 t+i+j-2}
$$

where $\delta_{i}$ for $i=1, \ldots, 6$ are constants depending on $t, i$ and $j$.

Lemma 3.2. The integral $F_{30}^{3}(r)$ is a polynomial in the variable $r$ of degree $\lambda_{3}$ equal to

$$
\begin{aligned}
\max & \{m+2[(k-1) / 2], \\
& m+2[(n-1) / 2]+2[m / 2]+2, \\
& m+2[m / 2]+2[k / 2]-2\}
\end{aligned}
$$

(For an explicit expression of the polynomial $F_{30}^{3}(r)$ we refer the reader to the proof of Lemma 3.2).
Proof. We first note that $\partial F_{2}(r, \theta) / \partial r$ is equal to

$$
\begin{aligned}
& \sum_{i=0}^{n}(i+1) c_{i} r^{i} \cos ^{i} \theta\left(1-\cos ^{2} \theta\right)+ \\
& \sum_{i=0}^{m} i d_{i, 2} r^{i-1} \cos ^{i} \theta \sin \theta+\sum_{i=0}^{k} i d_{i, 1} r^{i-1} \cos ^{i+1} \theta- \\
& \sum_{i=0}^{[(n-1) / 2]} \sum_{j=0}^{[(n-1) / 2]}(2 i+2 j+3) a_{2 i+1} a_{2 j+1} r^{2 i+2 j+2} . \\
& \cos ^{2 i+2 j+3} \sin ^{3} \theta-2 \sum_{i=0}^{[(n-1) / 2]} \sum_{j=0}^{m}(2 i+j+1) a_{2 i+1} \\
& b_{j, 2} r^{2 i+j} \cos ^{2 i+j+2} \theta \sin ^{2} \theta+ \\
& \sum_{i=0}^{n-1) / 2]} \sum_{j=0}^{[k / 2]}(2 i+2 j+1) a_{2 i+1} b_{2 j, 1} r^{2 i+2 j} . \\
& \cos ^{2 i+2 j+1} \theta \sin \theta\left(1-2 \cos ^{2} \theta\right)- \\
& \sum_{i=0}^{m} \sum_{j=0}^{m}(i+j-1) b_{i, 2} b_{j, 2} r^{i+j-2} \cos ^{i+j+1} \theta \sin \theta+ \\
& \sum_{i=0}^{m} \sum_{j=0}^{[k / 2]}(i+2 j-1) b_{i, 2} b_{2 j, 1} r^{i+2 j-2} \cos ^{i+2 j} \theta . \\
& \left(1-2 \cos ^{2} \theta\right)+\sum_{i=0}^{[k / 2]} \sum_{j=0}^{[k / 2]}(2 i+2 j-1) b_{2 i, 1} b_{2 j, 1} .
\end{aligned}
$$

Hence, using the formulae in the appendix and the explicit formula of $F_{30}^{3}(r)$ given in (13) we obtain that $F_{30}^{3}(r)$ is equal to

$$
\begin{aligned}
& \sum_{t=0}^{[(n-1) / 2]} \sum_{\substack{i=0 \\
[(n-1) / 2]}}^{[m / 2]} \rho_{1} a_{2 t+1} d_{2 i, 2} r^{2 t+2 i+1}+ \\
& \sum_{t=0}^{[m / 2]} \sum_{i=0}^{[(m-1) / 2]} \sum_{j=0}^{[m / 2]} \rho_{2} a_{2 t+1} b_{2 i, 2} b_{2 j+1,2} r^{2 t+2 i+2 j+1}+ \\
& \sum_{t=0}^{[m / 2]} \sum_{i=0}^{[k / 2]} \rho_{3} b_{2 t, 2} d_{2 i, 1} r^{2 t+2 i-1}+ \\
& \sum_{t=0}^{m} \sum_{i=0}^{[(k-1) / 2]} \rho_{4} b_{t, 2} d_{2 i+1,1} r^{t+2 i}+ \\
& \sum_{t=0}^{m} \sum_{i=0}^{[n / 2]} \rho_{5} b_{t, 2} c_{2 i} r^{t+2 i}+ \\
& \sum_{t=0}^{[m / 2]} \sum_{i=0}^{[(n-1) / 2]} \rho_{6} b_{2 t, 2} c_{2 i+1} r^{2 t+2 i+1}+ \\
& \sum_{t=0}^{m} \sum_{i=0}^{[(n-1) / 2]} \sum_{j=0}^{[m / 2]} \rho_{7} b_{t, 2} a_{2 i+1} b_{2 j, 2} r^{t+2 i+2 j+2}+
\end{aligned}
$$




$$
\begin{aligned}
& \sum_{t=0}^{[m / 2]} \sum_{i=0}^{[(n-1) / 2]} \sum_{j=0}^{[(m-1) / 2]} \rho_{8} b_{2 t, 2} a_{2 i+1} b_{2 j+1,2} r^{2 t+2 i+2 j+3}+ \\
& \sum_{t=0}^{m} \sum_{i=0}^{[m / 2]} \sum_{j=0}^{[k / 2]} \rho_{9} b_{t, 2} a_{2 i} b_{2 j+1,2} r^{t+2 i+2 j-2}+ \\
& \sum_{t=0}^{[m / 2]} \sum_{i=0}^{[(m-1) / 2]} \sum_{j=0}^{[k / 2]} \rho_{10} b_{2 t, 2} b_{2 i+1,2} b_{2 j, 1} r^{2 t+2 i+2 j-1}+ \\
& \sum_{t=0}^{[k / 2]} \sum_{i=0}^{[m / 2]} \rho_{11} b_{2 t, 1} d_{2 i, 2} r^{2 t+2 i-1}+ \\
& \sum_{t=0}^{[k / 2]} \sum_{i=0}^{[m / 2]} \sum_{j=0}^{[(m-1) / 2]} \rho_{12} b_{2 t, 1} b_{2 i, 2} b_{2 j+1,2} r^{2 t+2 i+2 j-1},
\end{aligned}
$$

where $\rho_{i}$ for $i=1, \ldots, 12$ are constants depending on $t, i$ and $j$.

Lemma 3.3. The integral $F_{30}^{4}(r)$ is a polynomial in the variable $r$ of degree $\lambda_{4}$ equal to

$$
\begin{aligned}
\max & \{2[n / 2]+2[(m-1) / 2]+1, \\
& 2[(m-1) / 2]+2[(k-1) / 2]+1, \\
& 2[(n-1) / 2]+2[m / 2]+2[(m-1) / 2]+1, \\
& 2[m / 2]+2[(m-1) / 2]+2[k / 2]-1\} .
\end{aligned}
$$

(For an explicit expression of the polynomial $F_{30}^{4}(r)$ we refer the reader to the proof of Lemma 3.3).

Proof. We will first compute in $F_{3}$ all the terms that have non-zero integral from 0 to $2 \pi$. To do it, we note that in view of (10) $F_{3}$ is equal to

$$
C-\frac{1}{r}\left(B A_{1}+A B_{1}\right)+\frac{1}{r^{2}} A A_{1}^{2} \text {. }
$$

Then

$$
\begin{array}{r}
\frac{1}{2 \pi} \int_{0}^{2 \pi} C(\theta, r) d \theta=\sum_{i=0}^{[n / 2]} \zeta_{1} p_{2 i} r^{2 i+1}+ \\
\sum_{i=0}^{[(k-1) / 2]} \zeta_{2} q_{2 i+1,1} r^{2 i+1}
\end{array}
$$

where the constants $\zeta_{1}, \zeta_{2}$ depend on $i$. Furthermore, using the formulae of the Appendix we conclude that

$$
\begin{aligned}
\frac{1}{2 \pi r} & \int_{00}^{2 \pi}\left(A B_{1}+B A_{1}\right)(\theta, r) d \theta= \\
& \sum_{i=0}^{[n / 2]} \sum_{j=0}^{[(m-1) / 2]} \zeta_{3} a_{2 i} d_{2 j+1,2} r^{2 i+2 j+1}+ \\
& \sum_{i=0}^{[(n-1) / 2]} \sum_{j=0}^{[m / 2]} \zeta_{4} a_{2 i+1} d_{2 j, 2} r^{2 i+2 j+1}+
\end{aligned}
$$

$$
\begin{aligned}
& \sum_{i=0}^{[m / 2]} \sum_{j=0}^{[(n-1) / 2]} \zeta_{5} b_{2 i, 2} c_{2 j+1} r^{2 i+2 j+1}+ \\
& \sum_{i=0}^{[(m-1) / 2]} \sum_{j=0}^{[n / 2]} \zeta_{6} b_{2 i+1,2} c_{2 j} r^{2 i+2 j+1}+ \\
& \sum_{i=0}^{[m / 2]} \sum_{j=0}^{[k / 2]} \zeta_{7} b_{2 i, 2} d_{2 j, 1} r^{2 i+2 j-1}+ \\
& \sum_{i=0}^{[(m-1) / 2]} \sum_{j=0}^{[(k-1) / 2]} \zeta_{8} b_{2 i+1,2} d_{2 j+1,1} r^{2 i+2 j+1}+ \\
& \sum_{i=0}^{[k / 2]} \sum_{j=0}^{[m / 2]} \zeta_{9} b_{2 i, 1} d_{2 j, 2} r^{2 i+2 j-1},
\end{aligned}
$$

where the constants $\zeta_{l}$ for $l=3, \ldots, 9$ depend on $i, j$. Finally, since $A_{1}^{2} / r^{2}$ is equal to

$$
\begin{aligned}
& \sum_{i=0}^{[(n-1) / 2]} \sum_{j=0}^{[(n-1) / 2]} a_{2 i+1} a_{2 j+1} r^{2 i+2 j+2} \cos ^{2 i+2 j+4} \theta . \\
& \left(1-\cos ^{2} \theta\right)+2 \sum_{i=0}^{[(n-1) / 2]} \sum_{j=0}^{m} a_{2 i+1} b_{j, 2} r^{2 i+j} . \\
& \cos ^{2 i+j+3} \theta \sin \theta-2 \sum_{i=0}^{[(n-1) / 2]} \sum_{j=0}^{[k / 2]} a_{2 i+1} b_{2 j, 1} r^{2 i+2 j} . \\
& \cos ^{2 i+2 j+2} \theta\left(1-\cos ^{2} \theta\right)+\sum_{i=0}^{m} \sum_{j=0}^{m} b_{i, 2} b_{j, 2} r^{i+j-2} . \\
& \cos ^{i+j+2} \theta-2 \sum_{i=0}^{m} \sum_{j=0}^{[k / 2]} b_{i, 2} b_{2 j, 1} r^{i+2 j-2} \cos ^{i+2 j+1} \theta . \\
& \sin \theta+\sum_{i=0}^{[k / 2]} \sum_{j=0}^{[k / 2]} b_{2 i, 1} b_{2 j, 1} r^{2 i+2 j-2} \cos ^{2 i+2 j} \theta . \\
& \left(1-\cos ^{2} \theta\right),
\end{aligned}
$$

using the formulae of the Appendix we conclude that

$$
\begin{aligned}
& \frac{1}{2 \pi r^{2}} \int_{0}^{2 \pi}\left(A A_{1}^{2}\right)(\theta, r) d \theta= \\
& \sum_{t=0}^{[(n-1) / 2]} \sum_{i=0}^{[m / 2]} \sum_{j=0}^{[(m-1) / 2]} \zeta_{10} a_{2 t+1} b_{2 i, 2} b_{2 j+1,2} r^{2 t+2 i+2 j+1}+ \\
& \sum_{t=0}^{[m / 2]} \sum_{i=0}^{[(n-1) / 2]} \sum_{j=0}^{[m-1) / 2]} \zeta_{11} b_{2 t, 2} a_{2 i+1} b_{2 j+1,2} r^{2 t+2 i+2 j+1}+ \\
& \sum_{t=0}^{[m / 2]} \sum_{i=0}^{[m-1) / 2]} \sum_{j=0}^{[k / 2]} \zeta_{12} b_{2 t, 2} b_{2 i+1,2} b_{2 j, 1} r^{2 t+2 i+2 j-1}+ \\
& \sum_{t=0}^{[m / 2]} \sum_{i=0}^{[(m-1) / 2]} \sum_{j=0}^{[m / 2} \zeta_{13} b_{2 t, 1} b_{2 i, 2} b_{2 j+1,2} r^{2 t+2 i+2 j-1},
\end{aligned}
$$


where the constants $\zeta_{l}$ for $l=10, \ldots, 13$ depend on $t, i, j$.

Lemma 3.4. The integral $F_{30}^{2}(r)$ is a polynomial in the variable $r$ of degree $\lambda_{5}$ equal to

$$
\begin{aligned}
\max & \{m+2[(k-1) / 2], \\
& m+2[(n-1) / 2]+2[m / 2], \\
& m+2[m / 2]+2[k / 2]-2, \\
& 4[(n-1) / 2]+2[(m-1) / 2]+1\} .
\end{aligned}
$$

(For an explicit expression of the polynomial $F_{30}^{2}(r)$ we refer the reader to the proof of Lemma 3.4).

Proof. We note that $F_{30}^{2}(r)$ is equal to

$$
\begin{aligned}
& \frac{1}{2 \pi} \int_{0}^{2 \pi} \frac{\partial F_{1}}{\partial r}(\theta, r) \int_{0}^{\theta} F_{2}(\psi, r) d \psi d \theta+ \\
& \frac{1}{2 \pi} \int_{0}^{2 \pi} \frac{\partial F_{1}}{\partial r}(\theta, r) \int_{0}^{\theta} \frac{\partial F_{1}}{\partial r}(\psi, r) y_{1}(\psi, r) d \psi d \theta .
\end{aligned}
$$

We will first compute the terms in

$$
\hat{F}_{30}^{2}(r)=\frac{1}{2 \pi} \int_{0}^{2 \pi} \frac{\partial F_{1}}{\partial r}(\theta, r) \int_{0}^{\theta} F_{2}(\psi, r) d \psi d \theta .
$$

For this we note that $\int_{0}^{\theta} F_{2}(\psi, r) d \psi$ is equal to

$$
\begin{aligned}
& \sum_{i=0}^{[n / 2]} c_{2 i} r^{2 i+1}\left(\beta_{i, 0} \theta+\sum_{s=1}^{i} \beta_{i, s} \sin (2 s \theta)\right)+ \\
& \sum_{i=0}^{[(n-1) / 2]} c_{2 i+1} r^{2 i+2} \sum_{s=0}^{i} \gamma_{i, s} \sin ((2 s+1) \theta)+ \\
& \sum_{i=0}^{m} \frac{d_{i, 2}}{i+1} r^{i}\left(1-\cos ^{i+1} \theta\right)+ \\
& \sum_{i=0}^{[k / 2]} d_{2 i, 1} r^{2 i} \sum_{s=0}^{i} \gamma_{i, s} \sin ((2 s+1) \theta)+ \\
& \sum_{i=0}^{[(k-1) / 2]} d_{2 i+1,1} r^{2 i+1}\left(\beta_{i, 0} \theta+\sum_{s=1}^{i+1} \beta_{i, s} \sin (2 s \theta)\right)- \\
& \sum_{i=0}^{[(n-1) / 2]} \sum_{j=0}^{[(n-1) / 2]} a_{2 i+1} a_{2 j+1} r^{2 i+2 j+3} . \\
& \left(\frac{1-\cos ^{2 i+2 j+4} \theta}{2 i+2 j+4}-\frac{1-\cos ^{2 i+2 j+6} \theta}{2 i+2 j+6}\right)- \\
& 2 \sum_{i=0}^{[(n-1) / 2][(m-1) / 2]} \sum_{j=0}^{i+j+2} a_{2 i+1} b_{2 j+1,2} r^{2 i+2 j+2} . \\
& \sum_{s=0}^{\gamma_{i+j+2, s}^{*} \sin ((2 s+1) \theta)-}
\end{aligned}
$$

$2 \sum_{i=0}^{[(n-1) / 2]} \sum_{j=0}^{[m / 2]} a_{2 i+1} b_{2 j, 2} r^{2 i+2 j+1}\left(\beta_{i+j+2,0}^{*} \theta+\right.$ $\left.\sum_{s=1}^{i+j+2} \beta_{i+j+2, s}^{*} \sin (2 s \theta)\right)+$

$\sum_{i=0}^{[(n-1) / 2]} \sum_{j=0}^{[k / 2]} a_{2 i+1} b_{2 j, 1} r^{2 i+2 j+1}\left(\frac{1-\cos ^{2 i+2 j+2} \theta}{2 i+2 j+2}-\right.$

$\left.\frac{1-\cos ^{2 i+2 j+4} \theta}{2 i+2 j+4}\right)-$

$\sum_{i=0}^{m} \sum_{j=0}^{m} b_{i, 2} b_{j, 2} r^{i+j-1} \frac{1-\cos ^{i+j+2} \theta}{i+j+2}+$

$\sum_{i=0}^{[(m-1) / 2]} \sum_{j=0}^{[k / 2]} b_{2 i+1,2} b_{2 j, 1} r^{2 i+2 j} \sum_{s=0}^{i+j+1} \bar{\gamma}_{i+j+1, s}$.

$\sin ((2 s+1) \theta)+\sum_{i=0}^{[m / 2]} \sum_{j=0}^{[k / 2]} b_{2 i, 2} b_{2 j, 1} r^{2 i+2 j-1}$.

$\left(\bar{\beta}_{i+j+1,0} \theta+\sum_{s=1}^{i+j+1} \bar{\beta}_{i+j+1, s} \sin (2 s \theta)\right)+$

$\sum_{i=0}^{[k / 2]} \sum_{j=0}^{[k / 2]} b_{2 i, 1} b_{2 j, 1} r^{2 i+2 j-1} \frac{1-\cos ^{2 i+2 j+2} \theta}{2 i+2 j+2}$.

Now using the non-zero formulae in the appendix we conclude that $\hat{F}_{30}^{2}(r)$ is equal to

$\sum_{\substack{t=0 \\[(n-1) / 2]}}^{[(m-1) / 2]} \sum_{\substack{i=0 \\[m / 2]}}^{[m / 2]} \mu_{1} a_{2 t} d_{2 i, 2} r^{2 t+2 i+1}+$

$\sum_{t=0} \sum_{i=0} \sum_{j=0} \mu_{2} a_{2 t+1} b_{2 i, 2} b_{2 j+1,2} r^{2 t+2 i+2 j+1}+$

$\sum_{t=0}^{m} \sum_{i=0}^{[n / 2]} \mu_{3} b_{t, 2} c_{2 i} r^{t+2 i}+\sum_{t=0}^{[m / 2]} \sum_{i=0}^{[(n-1) / 2]} \mu_{4} b_{2 t, 2} c_{2 i+1}$.

$r^{2 t+2 i+1}+\sum_{t=0}^{[m / 2]} \sum_{i=0}^{[k / 2]} \mu_{5} b_{2 t, 2} d_{2 i, 1} r^{2 t+2 i-1}+$

$\sum_{t=0}^{m} \sum_{i=0}^{[(k-1) / 2]} \mu_{6} b_{t, 2} d_{2 i+1,1} r^{t+2 i}+$

$[m / 2][(n-1) / 2][(m-1) / 2]$

$\sum_{t=0} \sum_{i=0} \sum_{j=0} \mu_{7} b_{2 t, 2} a_{2 i+1} b_{2 j+1,2} r^{2 t+2 i+2 j+1}+$

$\sum_{t=0}^{m} \sum_{i=0}^{[(n-1) / 2]} \sum_{j=0}^{[m / 2]} \mu_{8} b_{t, 2} a_{2 i+1} b_{2 j, 2} r^{t+2 i+2 j}+$

$\sum_{t=0}^{[m / 2]} \sum_{i=0}^{[(m-1) / 2]} \sum_{j=0}^{[k / 2]} \mu_{9} b_{2 t, 2} b_{2 i+1,2} b_{2 j, 1} r^{2 t+2 i+2 j-1}+$ 
$\sum_{t=0}^{m} \sum_{i=0}^{[m / 2]} \sum_{j=0}^{[k / 2]} \mu_{10} b_{t, 2} b_{2 i, 2} b_{2 j, 1} r^{t+2 i+2 j-2}+$

$[k / 2][m / 2]$

$\sum_{t=0}^{[k / 2]} \sum_{i=0}^{m / 2]} \mu_{11} b_{2 t, 1} d_{2 i, 2} r^{2 t+2 i-1}+$

$\sum_{t=0}^{[k / 2]} \sum_{i=0}^{[m / 2]} \sum_{j=0}^{[(m-1) / 2]} \mu_{12} b_{2 t, 1} b_{2 i, 2} b_{2 j+1,2} r^{2 t+2 i+2 j-1}$

where the constants $\mu_{i}$ for $i=1, \ldots, 12$ depend on $t, i, j$.

Now we compute $\int_{0}^{\theta} y_{1}(r, \psi) \frac{\partial F_{1}}{\partial r}(r, \psi) d \psi$. We first note that $y_{1}(r, \psi) \frac{\partial F_{1}}{\partial r}(r, \psi)$ is equal to

$$
\begin{aligned}
& \sum_{i=0}^{[(n-1) / 2]} \sum_{j=0}^{[(n-1) / 2]} a_{2 i+1}(2 j+2) a_{2 j+1} r^{2 i+2 j+3} . \\
& \sum_{\substack{i+1 \\
\text { [n }}}^{[(n-1) / 2]} \tilde{\gamma}_{i, s} \cos ^{2 j+1} \psi\left(1-\cos ^{2} \psi\right) \sin ((2 s+1) \psi)+ \\
& \sum_{i=0}^{m} \sum_{j=0}^{m} a_{2 i+1} j b_{j, 2} r^{2 i+j+1} \sum_{s=0}^{i+1} \tilde{\gamma}_{i, s} \cos ^{j} \psi
\end{aligned}
$$$$
\sin \psi \sin ((2 s+1) \psi)+\sum_{i=0}^{[(n-1) / 2]} \sum_{j=0}^{[k / 2]} a_{2 i+1} 2 j b_{2 j, 1} .
$$$$
r^{2 i+2 j+1} \sum_{s=0}^{i+1} \tilde{\gamma}_{i, s} \cos ^{2 j+1} \psi \sin ((2 s+1) \psi)+
$$$$
\sum_{i=0}^{m} \sum_{j=0}^{[(n-1) / 2]} \frac{b_{i, 2}}{i+1}(2 j+2) a_{2 j+1} r^{i+2 j+1}\left(1-\cos ^{i+1} \psi\right) \text {. }
$$$$
\cos ^{2 j+1} \psi\left(1-\cos ^{2} \psi\right)+\sum_{i=0}^{m} \sum_{j=0}^{m} \frac{b_{i, 2}}{i+1} j b_{j, 2} r^{i+j-1} .
$$$$
\left(1-\cos ^{i+1} \psi\right) \cos ^{j} \psi \sin \psi+\sum_{i=0}^{m} \sum_{j=0}^{[k / 2]} \frac{b_{i, 2}}{i+1} 2 j b_{2 j, 1} .
$$$$
r^{i+2 j-1}\left(1-\cos ^{i+1} \psi\right) \cos ^{2 j+1} \psi+\sum_{i=0}^{[k / 2]} \sum_{j=0}^{[(n-1) / 2]} b_{2 i, 1} \text {. }
$$$$
(2 j+2) a_{2 j+1} r^{2 i+2 j+1} \sum_{s=0}^{i} \gamma_{i, s} \cos ^{2 j+1} \psi\left(1-\cos ^{2} \psi\right) \text {. }
$$$$
\sin ((2 s+1) \psi)+\sum_{i=0}^{[k / 2]} \sum_{j=0}^{m} b_{2 i, 1} j b_{j, 2} r^{2 i+j-1} \text {. }
$$$$
\sum_{s=0}^{i} \gamma_{i, s} \cos ^{j} \psi \sin \psi \sin ((2 s+1) \psi)+
$$$$
\sum_{i=0}^{[k / 2]} \sum_{j=0}^{[k / 2]} b_{2 i, 1} 2 j b_{2 j, 1} r^{2 i+2 j-1} \sum_{s=0}^{i} \gamma_{i, s} \cos ^{2 j+1} \psi \text {. }
$$

$$
\sin ((2 s+1) \psi) \text {. }
$$

Hence using the formulae in the appendix we deduce that $\int_{0}^{\theta} y_{1}(r, \psi) \frac{\partial F_{1}}{\partial r}(r, \psi) d \psi$ is equal to $\sum_{i=0}^{[(n-1) / 2]} \sum_{\substack{j=0 \\ j+s+2}}^{[(n-1) / 2]} a_{2 i+1,2}(2 j+2) a_{2 j+1} r^{2 i+2 j+3}$. $\sum_{\substack{s=0 \\[(n-1) / 2]}}^{i+1} \tilde{\gamma}_{i, s} \sum_{\substack{r=1 \\[(m-1) / 2]}}^{j+s+2} \hat{R}_{j+s+2, r} \cos (2 r \theta)+$ $\sum_{i=0}^{[(n-1) / 2]} \sum_{j=0}^{r(m-1) / 2]} a_{2 i+1}(2 j+1) b_{2 j+1,2} r^{2 i+2 j+2}$. $\sum_{s=0}^{i+1} \tilde{\gamma}_{i, s} \sum_{r=1}^{j+s+1} U_{j+s+1, r} \sin ((2 r+1) \theta)+$ $\sum_{i=0}^{[(n-1) / 2]} \sum_{j=0}^{[m / 2]} a_{2 i+1} 2 j b_{2 j, 2} r^{2 i+2 j+1} \sum_{s=0}^{i+1} \tilde{\gamma}_{i, s}$. $\left(W_{j+s+1} \theta+\sum_{r=1}^{j+s+1} W_{j+s+1, r} \sin (2 r \theta)\right)+$ $[(n-1) / 2][k / 2]$ $\sum_{i=0} \sum_{j=0} a_{2 i+1} 2 j b_{2 j, 1} r^{2 i+2 j+1}$. $\sum_{s=0}^{i+1} \tilde{\gamma}_{i, s} \sum_{r=1}^{j+s+1} R_{j+s, r} \cos (2 r \theta)+$ $\sum_{i=0}^{[m / 2]} \sum_{j=0}^{[(n-1) / 2]} \frac{b_{2 i, 2}}{2 i+1}(2 j+2) a_{2 j+1} r^{2 i+2 j+1}$. $\left(\beta_{i+j+2}^{*} \theta+\sum_{r=1}^{i+j+2} \beta_{i+j+2, r}^{*} \sin (2 r \theta)\right)+$ $[(m-1) / 2][(n-1) / 2]$ $\sum_{i=0}^{[(m-1) / 2]} \sum_{j=0}^{[(n-1) / 2]} \frac{b_{2 i+1,2}}{2 i+2}(2 j+2) a_{2 j+1} r^{2 i+2 j+2}$. $\sum_{m}^{i+j+2} \gamma_{i+j+2, r}^{*} \sin ((2 r+1) \theta)+$ $\sum_{i=0}^{m} \sum_{j=0}^{m} \frac{b_{i, 2}}{i+1} j b_{j, 2} r^{i+j-1}\left(\frac{1-\cos ^{j+1} \theta}{j+1}-\right.$ $\left.\frac{1-\cos ^{i+j+2} \theta}{i+j+2}\right)+\sum_{i=0}^{[m / 2]} \sum_{j=0}^{[k / 2]} \frac{b_{2 i, 2}}{2 i+1} 2 j b_{2 j, 1} r^{2 i+2 j-1}$. $\left(\bar{\beta}_{i+j+1} \theta+\sum_{r=1}^{i+j+1} \bar{\beta}_{i+j+1, r} \sin (2 r \theta)\right)+$ $\sum_{i=0}^{[(m-1) / 2]} \sum_{j=0}^{[k / 2]} \frac{b_{2 i+1,2}}{2 i+2} 2 j b_{2 j, 1} r^{2 i+2 j} \sum_{r=1}^{i+j+1} \bar{\gamma}_{i+j+1, r}$ $\sin ((2 r+1) \theta)+\sum_{i=0}^{[k / 2]} \sum_{j=0}^{[(n-1) / 2]} b_{2 i, 1}(2 j+2) a_{2 j+1}$. $r^{2 i+2 j+1} \sum_{s=0}^{i+1} \gamma_{i, s} \sum_{r=1}^{j+s+2} \hat{R}_{j+s+2, r} \cos (2 r \theta)+$ 


$$
\begin{aligned}
& \sum_{i=0}^{[k / 2]} \sum_{j=0}^{[m / 2]} b_{2 i, 1} 2 j b_{2 j, 2} r^{2 i+2 j-1} \sum_{s=0}^{i+1} \gamma_{i, s}\left(W_{j+s+1} \theta+\right. \\
& \left.\sum_{r=1}^{j+s+1} W_{j+s+1, r} \sin (2 r \theta)\right)+\sum_{i=0}^{[k / 2]} \sum_{j=0}^{[(m-1) / 2]} b_{2 i, 1} \\
& (2 j+1) b_{2 j+1,2} r^{2 i+2 j} \sum_{s=0}^{i+1} \gamma_{i, s} \sum_{r=1}^{j+s+1} U_{j+s+1, r} \\
& \sin ((2 r+1) \theta)+\sum_{i=0}^{[k / 2]} \sum_{j=0}^{[k / 2]} b_{2 i, 1} 2 j b_{2 j, 1} r^{2 i+2 j-1} . \\
& \sum_{s=0}^{i+1} \gamma_{i, s} \sum_{r=1}^{j+s+1} R_{j+s, r} \cos (2 r \theta) .
\end{aligned}
$$

Hence using the zero formulae in the appendix we compute $\bar{F}_{30}^{2}(r)$ and we obtain that it is equal to

$$
\frac{1}{2 \pi} \int_{0}^{2 \pi} \frac{\partial F_{1}}{\partial r}(\theta, r) \int_{0}^{\theta} \frac{\partial F_{1}}{\partial r}(\psi, r) y_{1}(\psi, r) d \psi d \theta,
$$

and this integral is

$$
\begin{aligned}
& \sum_{t=0}^{[(n-1) / 2]} \sum_{i=0}^{m} \sum_{j=0}^{[m / 2]} \nu_{1} a_{2 t+1} b_{i, 2} b_{2 j, 2} r^{2 t+i+2 j}+ \\
& {[(n-1) / 2][m / 2][(m-1) / 2]} \\
& \sum_{t=0} \sum_{i=0} \sum_{j=0} \nu_{2} a_{2 t+1} b_{2 i, 2} b_{2 j+1,2} r^{2 t+2 i+2 j+1}+ \\
& {[m / 2][(n-1) / 2][(m-1) / 2]} \\
& \sum_{t=0} \sum_{i=0} \sum_{j=0} \nu_{3} b_{2 t, 2} a_{2 i+1} b_{2 j+1,2} r^{2 t+2 i+2 j+1}+ \\
& \sum_{t=0}^{m} \sum_{i=0}^{[(n-1) / 2]} \sum_{j=0}^{[m / 2]} \nu_{4} b_{t, 2} a_{2 i+1} b_{2 j, 2} r^{t+2 i+2 j}+ \\
& \sum_{t=0}^{m} \sum_{i=0}^{[m / 2]} \sum_{j=0}^{[(n-1) / 2]} \nu_{5} b_{t, 2} b_{2 i, 2} a_{2 j+1} r^{t+2 i+2 j}+ \\
& \sum_{t=0}^{[m / 2]} \sum_{i=0}^{[(m-1) / 2]} \sum_{j=0}^{[(n-1) / 2]} \nu_{6} b_{2 t, 2} b_{2 i+1,2} a_{2 j+1} r^{2 t+2 i+2 j+1}+ \\
& \sum_{t=0}^{m} \sum_{i=0}^{[m / 2]} \sum_{j=0}^{[k / 2]} \nu_{7} b_{t, 2} b_{2 i, 2} b_{2 j, 1} r^{t+2 i+2 j-2}+ \\
& \sum_{t=0}^{[m / 2]} \sum_{i=0}^{[(m-1) / 2]} \sum_{j=0}^{[k / 2]} \nu_{8} b_{2 t, 2} b_{2 i+1,2} b_{2 j, 1} r^{2 t+2 i+2 j-1}+ \\
& \sum_{t=0}^{m} \sum_{i=0}^{[k / 2]} \sum_{j=0}^{[m / 2]} \nu_{9} b_{t, 2} b_{2 i, 1} b_{2 j, 2} r^{t+2 i+2 j-2}+ \\
& \sum_{t=0}^{[m / 2]} \sum_{i=0}^{[k / 2]} \sum_{j=0}^{[(m-1) / 2]} \nu_{10} b_{2 t, 2} b_{2 i, 1} b_{2 j+1,2} r^{2 t+2 i+2 j-1}+
\end{aligned}
$$

$$
\begin{aligned}
& \sum_{t=0}^{[k / 2]} \sum_{i=0}^{m} \sum_{j=0}^{[m / 2]} \nu_{11} b_{2 t, 1} b_{i, 2} b_{2 j, 2} r^{2 t+i+2 j-2}+ \\
& \sum_{t=0}^{[k / 2]} \sum_{i=0}^{[m / 2]} \sum_{j=0}^{[(m-1) / 2]} \nu_{12} b_{2 t, 1} b_{2 i, 2} b_{2 j+1,2} r^{2 t+2 i+2 j-1},
\end{aligned}
$$

where the constants $\nu_{i}$ for $i=1, \ldots, 12$ depend on $t, i, j$.

\section{Appendix: Formulae}

In this appendix we recall some formulae that will be used during the paper, see for more details [Abramowitz \& Stegun, 1964]. For $i \geq 0$ we have

$$
\begin{gathered}
\int_{0}^{2 \pi} \cos ^{2 i+1} \theta \sin ^{2} \theta d \theta=\int_{0}^{2 \pi} \cos ^{i} \theta \sin \theta d \theta= \\
\int_{0}^{2 \pi} \cos ^{2 i+1} \theta d \theta=0 \\
\frac{1}{2 \pi} \int_{0}^{2 \pi} \cos ^{2 i} \theta d \theta=\alpha_{i}
\end{gathered}
$$

where $\alpha_{i}$ is a non-zero constant,

$$
\begin{array}{r}
\int_{0}^{\theta} \cos ^{2 i} \phi d \phi=\frac{1}{2^{2 i}}\left(\begin{array}{c}
2 i \\
i
\end{array}\right) \theta+\frac{1}{2^{2 i}} \sum_{l=1}^{i}\left(\begin{array}{c}
2 i \\
i+1
\end{array}\right) . \\
\frac{1}{l} \sin (2 l \theta)=\beta_{i, 0} \theta+\sum_{l=1}^{i} \beta_{i, l} \sin (2 l \theta),
\end{array}
$$

$$
\begin{aligned}
\int_{0}^{\theta} \cos ^{2 i+1} \phi d \phi=\frac{1}{2^{2 i}} \sum_{l=0}^{i}\left(\begin{array}{c}
2 i+1 \\
i-1
\end{array}\right) \frac{1}{2 l+1} . \\
\sin ((2 l+1) \theta)=\sum_{l=0}^{i} \gamma_{i, l} \sin ((2 l+1) \theta),
\end{aligned}
$$$$
\int_{0}^{\theta} \cos ^{i} \phi \sin \phi d \phi=\frac{1}{i+1}\left(1-\cos ^{i+1} \theta\right),
$$$$
\int_{0}^{\theta} \cos ^{2 i} \psi \sin ((2 s+1) \psi) d \psi=\sum_{r=1}^{i+s} P_{i+s, r} \cos ((2 r+1) \theta),
$$$$
\int_{0}^{\theta} \cos ^{2 i} \psi \sin (2 s \psi) d \psi=\sum_{r=1}^{i+s} Q_{i+s, r} \cos (2 r \theta),
$$$$
\int_{0}^{\theta} \cos ^{2 i+1} \psi \sin ((2 s+1) \psi) d \psi=\sum_{r=1}^{i+s+1} R_{i+s, r} \cos (2 r \theta),
$$

$\int_{0}^{\theta} \cos ^{2 i+1} \psi \sin (2 s \psi) d \psi=\sum_{r=1}^{i+s} T_{i+s, r} \cos ((2 r+1) \theta)$, 


$$
\begin{aligned}
& \int_{0}^{\theta} \cos ^{2 i+1} \psi \sin \psi \sin ((2 s+1) \psi) d \psi= \\
& \sum_{r=1}^{i+s+1} U_{i+s+1, r} \sin ((2 r+1) \theta) \\
& \int_{0}^{\theta} \cos ^{2 i+1} \psi \sin \psi \sin (2 s \psi) d \psi= \\
& V_{i+s+1} \theta+\sum_{r=1}^{i+s+1} V_{i+s+1, r} \sin (2 r \theta), \\
& \int_{0}^{\theta} \cos ^{2 i} \psi \sin \psi \sin ((2 s+1) \psi) d \psi= \\
& W_{i+s+1} \theta+\sum_{r=1}^{i+s+1} W_{i+s+1, r} \sin (2 r \theta), \\
& \int_{0}^{\theta} \cos ^{2 i} \psi \sin \psi \sin (2 s \psi) d \psi= \\
& \sum_{r=1}^{i+s} Z_{i+s, r} \sin ((2 r+1) \theta) \\
& \frac{1}{2 \pi} \int_{0}^{2 \pi} \cos ^{2 i+1} \theta \sin \theta \sin (2 l \theta) d \theta=K_{i, l}, \quad l \geq 1 \\
& \int_{0}^{2 \pi} \cos ^{i} \theta \sin \theta \sin (r \theta) \sin (s \theta) d \theta=0, \quad r, s \in \mathbb{N} .
\end{aligned}
$$$$
\int_{0}^{2 \pi} \cos ^{i} \theta \sin \theta \cos (j \theta) d \theta=
$$$$
\int_{0}^{2 \pi} \cos ^{2 i} \theta \cos ((2 j+1) \theta) d \theta=
$$$$
\int_{0}^{2 \pi} \cos ^{2 i+1} \theta \cos (2 j \theta) d \theta=0, j \in \mathbb{Z}
$$$$
\int_{0}^{2 \pi} \cos ^{2 i} \theta \cos (2 j \theta) d \theta=D_{i, j}
$$$$
\int_{0}^{2 \pi} \cos ^{2 i+1} \theta \cos ((2 j+1) \theta) d \theta=E_{i, j},
$$

where $D_{i, j}, E_{i, j}$ are nonzero constants,

$$
\int_{0}^{2 \pi} \theta \cos ^{2 i} \theta d \theta=F_{i}, \quad \int_{0}^{2 \pi} \theta \cos ^{i} \theta \sin \theta d \theta=G_{i}
$$

where $F_{i}, G_{i}$ are nonzero constants,

$$
\begin{gathered}
\int_{0}^{2 \pi} \cos ^{2 i+1} \theta \sin \theta \sin ((2 l+1) \theta) d \theta= \\
\int_{0}^{2 \pi} \cos ^{2 i} \theta \sin \theta \sin (2 l \theta) d \theta=0, \quad l \geq 0 \\
\int_{0}^{2 \pi} \cos ^{i} \theta \sin ((2 l+1) \theta) d \theta= \\
\int_{0}^{2 \pi} \cos ^{i} \theta \sin (2 l \theta) d \theta=0, \quad l \geq 0
\end{gathered}
$$

In the remainder formulae $r, s \in \mathbb{Z}$ :

$$
\begin{gathered}
\int_{0}^{2 \pi} \cos ^{2 i+1} \theta \sin ((2 r+1) \theta) \sin ((2 s+1) \theta) d \theta=0, \\
\int_{0}^{2 \pi} \cos ^{2 i+1} \theta \sin (2 r \theta) \sin (2 s \theta) d \theta=0, \\
\frac{1}{2 \pi} \int_{0}^{2 \pi} \cos ^{2 i} \theta \sin ((2 r+1) \theta) \sin (2 s \theta) d \theta=0, \\
\frac{1}{2 \pi} \int_{0}^{2 \pi} \cos ^{2 i} \theta \sin ((2 r+1) \theta) \sin ((2 s+1) \theta) d \theta=\Delta_{i, r, s}, \\
\int_{0}^{2 \pi} \cos ^{2 i+1} \theta \sin \left((2 r \theta) \sin (2 s \theta) d \theta=\Gamma_{i, r, s},\right.
\end{gathered}
$$

where $\Delta_{i, r, s}, \Gamma_{i, r, s}$ and $\Upsilon_{i, r, s}$ are non-zero constants.

\section{Acknowledgments}

The first author has been supported by the grants MICINN/FEDER MTM 2009-03437, AGAUR 2009SGR 410 and ICREA Academia. The second author has been supported by the grant AGAUR PIV-DGR-2010 and by FCT through CAMGSD. 


\section{References}

Abramowitz, M., \& Stegun, I., Handbook of mathematical functions with formulas, graphs, and mathematical tables, National Bureau of Standards Applied Mathematics Series 55, Washington.

Alavez-Ramirez, J., Blé, G., Llibre, J. \& and LopezLopez, J., [2012] "On the maximum number of limit cycles of a class of generalized Liénard differential systems," to appear in Int. J. Bifurcation and Chaos, .

Blows, T.R., \& Lloyd, N.G., [1984] "The number of small-amplitude limit cycles of Liénard equations," Math. Proc. Camb. Phil. Soc. 95, 359366.

Buică, A., \& Llibre, J., [2004] "Averaging methods for finding periodic orbits via Brouwer degree," Bull. Sci. Math. 128, 7-22.

Christopher, C.J., \& Lynch, S., [1999] "Smallamplitude limit cycle bifurcations for Liénard systems with quadratic or cubic dapimg or restoring forces," Nonlinearity 12, 1099-1112.

Coppel, W.A., [1998] "Some quadratic systems with at most one limit cycles," Dynamics Reported 2, $61-68$.

De Maesschalck, P., \& Dumortier, F., [2011] "Classical Liénard equation of degree $n \geq 6$ can have $\left[\frac{n-1}{2}\right]+2$ limit cycles," J. Differential Equations 250, 2162-2176.

Dumortier, F., Panazzolo, D., \& Roussarie, R., [2007] "More limit cycles than expected in Liénard systems," Proc. Amer. Math. Soc. 135, 1895-1904.

Dumortier,F., \& Li, C., [1996] "On the uniqueness of limit cycles surrounding one or more singularities for Liénard equations," Nonlinearity 9, 1489-1500.

Dumortier,F., \& Li, C., [1997] "Quadratic Liénard equations with quadratic damping," J. Differential Equations 139, 41-59.

Dumortier, F., \& Rousseau, C., [1990] "Cubic Liénard equations with linear dapimg," Nonlinearity 3, 1015-1039.
Gasull, A., \& Torregrosa,J., [1998] "SmallAmplitude limit cycles in Liénard systems via multiplicity," J. Differential Equations 159, 1015-1039.

Guckenheimer, J. \& Holmes, P., [1990] Nonlinear Osciallations, Dynamical Systems, and Bifurcations of Vector Fields, Revised and corrected reprint of the 1983 original, Applied Mathematical Sciences, Vol. 42, Springer-Verlag, New York.

Li, C., \& Llibre, J., [2012] "Uniqueness of limit cycles for Liénard differential equations of degree four," J. Differential Equations 252, 3142-3162.

Liénard, A., [1928] "Étude des oscillations entrenues," Revue Génerale de l'Électricité 23, 946-954.

Lins, A., de Melo, W., \& Pugh, C.C., [1977] "On Liénard's Equation," Lecture Notes in Math. 597, 335-357.

Llibre, J., [2002] "Averaging theory and limit cycles for quadratic systems," Radovi Matematicki 11, $215-228$.

Llibre, J., Mereu, A.C.,\& Teixeira, M.A. [2009] "Limit cycles of the generalized polynomial Liénard differential equations," Math. Proceed. Camb. Phyl. Soc. 148, 363-383.

Llibre, J., \& Valls, C., [2011] "On the number of limit cycles of a class of polynomial differential systems," preprint, .

Lloyd, N.G., [1988] "Limit cycles of polynomial systems - some recent developments," London Math. Soc. Lecture Note Ser. 127, 192-234.

Lloyd, N.G., \& Lynch, S., [1988] "Small-amplitude limit cycles of certain Liénard systems," Proc. Royal Soc. London Ser. A 418, 199-208.

Lynch, S., [1995] "Limit cycles if generalized Liénard equations," Applied Math. Letters 8, $15-17$.

Lynch, S., [1998] "Generalized quadratic Liénard equations," Applied Math. Letters 11, 7-10.

Lynch, S., [1999] "Generalized cubic Liénard equations," Applied Math. Letters 12, 1-6. 
Lynch, S., \& Christopher, C.J., [1999] "Limit cycles in highly non-linear differential equations," J. Sound Vib. 224, 505-517.

Rychkov, G.S., [1975] "The maximum number of limit cycle of the system $\dot{x}=y-a_{1} x^{3}-a_{2} x^{5}, \dot{y}=$ $-x$ is two," Differential'nye Uravneniya $\mathbf{1 1}$, 380-391.

Verhulst, F., [1991] Nonlinear Differential Equations and dynamical Systems Universitext, Springer-Verlag, New York.

Yu, P., \& Han, M., [2006] "Limit cycles in generalized Liénard systems," Chaos, Solitons and Fractals 30, 1048-1068. 